\title{
Running Coupling Constant from Position-Space Current-Current Correlation Functions in Three-Flavor Lattice QCD
}

\author{
Salvatore Calì ${ }^{1, *}$ Krzysztof Cichy, ${ }^{2, \dagger}$ Piotr Korcyl@ ${ }^{1,3, \ddagger}$ and Jakob Simeth $\oplus^{3, \S}$ \\ ${ }^{1}$ Institute of Theoretical Physics, Jagiellonian University, ulica Łojasiewicza 11, 30-348 Kraków, Poland \\ ${ }^{2}$ Faculty of Physics, Adam Mickiewicz University, ulica Uniwersytetu Poznańskiego 2, 61-614 Poznań, Poland \\ ${ }^{3}$ Institut für Theoretische Physik, Universität Regensburg, D-93040 Regensburg, Germany
}

(Received 17 March 2020; revised 30 September 2020; accepted 11 November 2020; published 9 December 2020)

\begin{abstract}
In this Letter, we provide a determination of the coupling constant in three-flavor quantum chromodynamics (QCD), $\alpha_{s}^{\overline{\mathrm{MS}}}(\mu)$, for $\overline{\mathrm{MS}}$ renormalization scales $\mu \in(1,2) \mathrm{GeV}$. The computation uses gauge field configuration ensembles with $\mathcal{O}(a)$-improved Wilson-clover fermions generated by the Coordinated Lattice Simulations (CLS) consortium. Our approach is based on current-current correlation functions and has never been applied before in this context. We convert the results perturbatively to the QCD $\Lambda$ parameter and obtain $\Lambda_{\frac{N_{f}}{\mathrm{MS}}}^{N^{2}}=342 \pm 17 \mathrm{MeV}$, which agrees with the world average published by the Particle Data Group and has competing precision. The latter was made possible by a unique combination of state-of-the-art CLS ensembles with very fine lattice spacings, further reduction of discretization effects from a dedicated numerical stochastic perturbation theory simulation, combining data from vector and axial-vector channels, and matching to high-order perturbation theory.
\end{abstract}

DOI: 10.1103/PhysRevLett.125.242002

Motivation.-The strength of strong interactions, parametrized by the scale-dependent coupling $\alpha_{s}$, typically quoted at the $Z$-boson pole mass, is one of the most important parameters of the standard model (SM). It is required in perturbative calculations in collider physics and its uncertainty is one of dominant sources of uncertainty in several SM predictions, as well as in tests of SM extensions [1]. Because of the non-Abelian Yang-Mills nature of quantum chromodynamics (QCD), $\alpha_{s}$ vanishes asymptotically at very high energies $[2,3]$ and experiments are able to follow this energy dependence in various processes over a wide range of energy scales. This allows us to determine $\alpha_{s}$ at several scales by fitting experimental data and matching to a perturbative expansion of an appropriate observable. Equivalently, using renormalization group concepts, one may parametrize the running of $\alpha_{s}$ by a single parameter, $\Lambda$, corresponding to the scale where perturbation theory breaks down. Examples of experimental processes for the extraction of the strong coupling or the $\Lambda$ parameter are hadronic $\tau$ decays, deep inelastic scattering, and hadronic final states of $e^{+} e^{-}$annihilation. For a review of many aspects of such determinations and the obtained values, see the Particle Data Group (PDG) review [4]. However, the strong coupling constant or the $\Lambda$ parameter can also be extracted

Published by the American Physical Society under the terms of the Creative Commons Attribution 4.0 International license. Further distribution of this work must maintain attribution to the author(s) and the published article's title, journal citation, and DOI. Funded by SCOAP. directly from the QCD Lagrangian, using the nonperturbative formulation of QCD on the lattice. This proceeds by calculating appropriately designed short-distance Euclidean observables and, again, matching them to their perturbative expansions. Over the years, several methods of how to design such observables have been proposed. Recent investigations employed, e.g., step scaling methods $[5,6]$, the static quark-antiquark potential [7-9], the vacuum polarization function [10], the heavy-quark current twopoint correlation function [11], QCD vertices (e.g., ghost gluon) [12], or eigenvalues of the lattice Dirac operator [13]. For a discussion and overview of these and older results, see the Flavor Lattice Averaging Group (FLAG) review [14]. The determinations from experiments and from the lattice enter the world average of $\alpha_{s}$ in the PDG review [4], recently with a visibly larger impact of lattice results due to their smaller total uncertainties.

In this Letter, we describe a novel method of estimating the running of the coupling or the $\Lambda$ parameter, using numerical simulations of QCD. The proposed method employs large volume simulations, it has a moderate numerical cost, and is clean and straightforward from the theoretical point of view. It is based on current-current correlation functions in position space, objects well studied and easily accessible in the lattice QCD framework. Thanks to the combination of very fine lattices generated by the Coordinated Lattice Simulations (CLS) effort [15,16], precise renormalization factors from the chirally rotated Schrödinger functional $(\chi \mathrm{SF})$ framework [17] and $\mathcal{O}(a)$-improvement coefficients [18,19], subtraction of leading-order and next-to-leading-order discretization 
TABLE I. Subset of $N_{f}=2+1$ CLS ensembles along the symmetric line $\kappa_{l}=\kappa_{s}$ used in this work $[15,16]$. The gauge action is treelevel Symanzik improved, while the fermionic one is the Wilson $O(a)$-improved (clover) action with the improvement coefficient, $c_{S W}$, determined nonperturbatively. rqcd30, X450, B450, X250, and X251 have been generated by the RQCD and Mainz Collaborations. For more details, see Refs. $[15,16]$. The values of $t_{0} / a^{2}$ are the reweighted estimates using the symmetric definition of the Yang-Mills action density [29]. The lattice spacings corresponding to different $\beta$ values are $0.07582(24) \mathrm{fm}(\beta=3.46), 0.0644(7) \mathrm{fm}(\beta=3.55), 0.0499$ (5) $\mathrm{fm}(\beta=3.7)$, and 0.0391(15) fm $(\beta=3.85)$ [29]. The scale setting is based on the determination of light hadron masses on all CLS ensembles with 6 lattice spacings from $\approx 0.1 \mathrm{fm}$ down to below $0.04 \mathrm{fm}$ and pion masses $\in[135,420] \mathrm{MeV}$. The Wilson flow scales are determined in the continuum using the $\Upsilon$ baryon mass as input. The last column indicates the number of configurations used.

\begin{tabular}{|c|c|c|c|c|c|}
\hline$\beta$ & Name & $\kappa_{l}=\kappa_{s}$ & $m_{\pi}[\mathrm{MeV}]$ & $t_{0} / a^{2}$ & \# conf \\
\hline 3.46 & B450 & 0.136890 & 419 & $3.663(11)$ & 320 \\
\hline 3.46 & $\operatorname{rqcd} 30$ & 0.136959 & 320 & $3.913(15)$ & 280 \\
\hline 3.46 & X4 50 & 0.136994 & 264 & $3.994(10)$ & 280 \\
\hline 3.55 & B250 & 0.136700 & 709 & $4.312(8)$ & 84 \\
\hline 3.55 & N202 & 0.137000 & 412 & $5.165(14)$ & 177 \\
\hline 3.55 & $\mathrm{X} 250$ & 0.137050 & 348 & $5.283(27)$ & 182 \\
\hline 3.55 & X251 & 0.137100 & 269 & $5.483(26)$ & 177 \\
\hline 3.7 & N303 & 0.136800 & 641 & $7.743(23)$ & 99 \\
\hline 3.7 & N300 & 0.137000 & 423 & $8.576(21)$ & 197 \\
\hline 3.85 & N500 & 0.13672514 & 599 & $12.912(75)$ & 100 \\
\hline 3.85 & $\mathrm{~J} 500$ & 0.136852 & 410 & $14.045(38)$ & 120 \\
\hline
\end{tabular}

effects estimated in the numerical stochastic perturbation theory (NSPT) formulation [20], and various improved analysis techniques, it yields a competitive total uncertainty. Therefore, it may serve as a robust method of estimating the $\Lambda$ parameter. The approach presented here is not limited to $\Lambda$ - a position-space analysis may also be used to reliably estimate other important observables, such as quark and gluon condensates [21], quark masses [22], or operator renormalization functions [23-26].

Strategy.-The strategy proposed in this Letter uses a combination of numerical lattice QCD calculations and high-order perturbative results. We concentrate on correlation functions of flavor nonsinglet bilinear quark operators of the form

$$
C_{\Gamma}\left(1 / x, m_{q}, a\right)=Z_{\Gamma}^{2}\left\langle\bar{\psi}^{i}(x) \Gamma \psi^{j}(x) \bar{\psi}^{j}(0) \Gamma \psi^{i}(0)\right\rangle,
$$

where $x$ is the physical distance, $m_{q}$ is the quark mass of three degenerate flavors of quarks, $a$ denotes the lattice spacing, $\Gamma=\left\{V \equiv \gamma_{\mu}, A \equiv \gamma_{\mu} \gamma_{5}\right\}, i \neq j$, and $Z_{\Gamma}$ is the (scale-independent) renormalization factor. For a reliable extraction of $\alpha_{s}$, we need to work in the regime of distances satisfying a window condition, $a \ll x \ll \Lambda^{-1}$. The former condition guarantees that discretization effects are not enhanced, while the latter establishes that reliable contact to perturbation theory can be made. After extrapolating the correlation functions to the continuum limit and after renormalization, they can be matched to their perturbative expansions in terms of $\alpha_{s}$, typically in the $\overline{\mathrm{MS}}$ scheme $\left(\alpha_{\overline{\mathrm{MS}}}(\mu)\right)$

$$
C_{\Gamma}(\mu)=c_{\Gamma}^{(1)} \alpha_{\overline{\mathrm{MS}}}(\mu)+c_{\Gamma}^{(2)} \alpha_{\overline{\mathrm{MS}}}^{2}(\mu)+\cdots
$$

where $C_{\Gamma}(\mu) \equiv C_{\Gamma}\left(1 / x, m_{q}=0, a=0\right)$. Such an expansion of current-current correlators is presently available up to four loops [27]. Knowing $C_{\Gamma}(\mu)$ from numerical simulations and the analytic form of coefficients $c_{\Gamma}^{(i)}$, we solve Eq. (2) for $\alpha_{\overline{\mathrm{MS}}}(\mu)$. Subsequently, we convert that value to our estimate of the $\Lambda$ parameter. Now, we provide details of the different steps needed to reliably obtain $C_{\Gamma}(\mu)$.

Crucial elements of the analysis. - We start with the bare lattice data for correlation functions $C_{A / V}\left(1 / x, m_{q}, a\right)$ with the $\mathcal{O}(a)$ improvement of the currents implemented by using improvement coefficients $c_{A}$ from Ref. [18] and $c_{V}$ from Ref. [19]. The ensembles used in this study are summarized in Table I. We perform 64 inexact and 2 exact measurements per configuration using the truncated solver method [28] and for every lattice distance $x / a$, we average correlators evaluated from all sites equivalent with respect to the hypercubic symmetry of the lattice.

At fixed lattice spacing and lattice distance, we extrapolate the correlators to the chiral limit. We use a fitting ansatz linear in the dimensionless combination $y=t_{0} m_{\pi}^{2}$, where $t_{0}$ is an intermediate unphysical scale introduced in Ref. [30] and we take the values of $t_{0} / a^{2}$ from Ref. [29]. The quality of the chiral fit was tested at $\beta=3.55$, where we have four pion masses available. We compared the linear fit in $y$ to either all ("lin4") or the three lightest masses ("lin3") with the quadratic one to all masses ("quad4") for all the relevant distances (for more details, see the Supplemental Material [31]). The small differences that we observe in the chiral limit amount, on average, to $0.17 \%$ (lin3 vs lin4) and $0.26 \%$ (quad4 vs lin4) at the level of correlators. Conservatively, we propagate the latter to $\alpha_{s}$ via a bootstrap procedure, taking for other $\beta$ values the linear ansatz. We denote the massless correlator by $C_{\Gamma}(1 / x, a)$. The massless correlators 
are then expressed in the $\overline{\mathrm{MS}}$ scheme, using renormalization factors calculated in Ref. [17], determined using the $\chi \mathrm{SF}$ framework [36].

A significant step to reliably perform the continuum limit extrapolation is to reduce the size of discretization effects present in the data. To this aim, we perturbatively compute $O\left(a^{\infty} g^{2}\right)$ artifacts, i.e., we replace the correlation functions

$$
C_{\Gamma}(1 / x, a) \rightarrow C_{\Gamma}(1 / x, a)+\left[C_{\Gamma}^{\text {cont }}(1 / x)-C_{\Gamma}^{\text {lat }}(a / x)\right],
$$

where $C_{\Gamma}^{t}=C_{\Gamma}^{\text {free, } t}+g_{0}^{2} C_{\Gamma}^{\text {one-loop }, t}, t \in\{$ cont, lat $\}$ and the superscript free/one-loop denotes the tree-level/one-loop contributions. The massless $\overline{\mathrm{MS}}$ continuum correlators, $C_{A / V}^{\text {cont }}$, are given in Ref. [27]. In turn, $C_{A / V}^{\text {lat }}$ are computed in NSPT [20] along the lines of Refs. [37,38] and are expressed in the $\overline{\mathrm{MS}}$ scheme using the renormalization factors for the employed gauge action [39]. (A more detailed description of the NSPT calculation will be presented in a separate publication [40]. For a shorter account, see the Supplemental Material.) Thus, all terms appearing on the rhs of Eq. (3) are correctly normalized correlators in the same scheme. The improved correlator, hence, has leading cutoff effects of $\mathcal{O}\left(a^{2} g^{4}\right)$. We demonstrate the reduction of discretization effects in Fig. 1, depicting the distance dependence of $C_{A}(1 / x, a)$ at $\beta=3.85$, for all points used in the extraction of $\alpha_{s}$. We show three data sets: without any correction, with the treelevel correction only, and with the full one-loop NSPT correction. The scatter of data points is clearly reduced, yielding a smooth curve. It is important to emphasize that the tree-level corrected data, even though seemingly already smooth, still prohibit any meaningful extraction

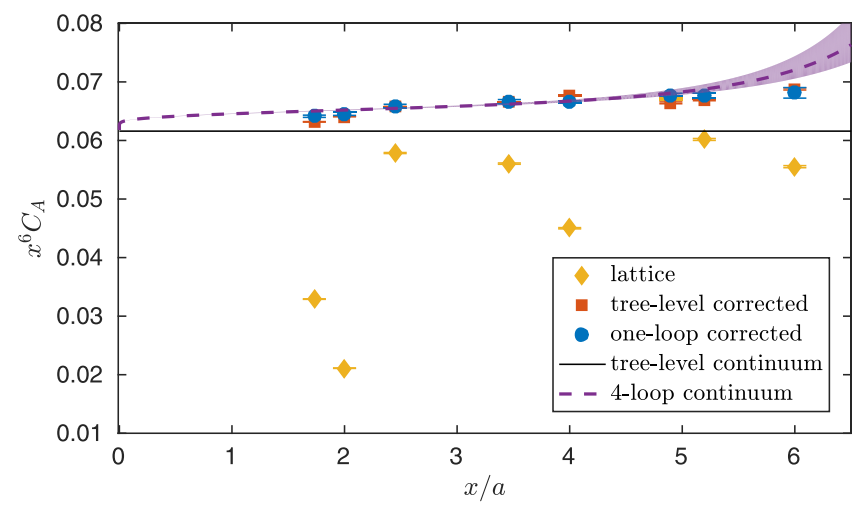

FIG. 1. Impact of the tree-level (red squares) and one-loop (blue circles) improvement of the massless axial current-current correlation function at $\beta=3.85$. The unimproved lattice correlators are shown as yellow rhombi. Tree-level and four-loop [27] continuum perturbative lines are also shown. The difference of the latter, corresponding to $\Lambda_{\overline{\mathrm{MS}}}^{N_{f}=3}$ found in this work, with respect to the blue points isolates the remaining discretization effects at this $\beta$. of $\alpha_{s}$ (see Supplemental Material for more details). Thus, reliable control of discretization effects necessitates the use of the one-loop subtraction of artifacts (all orders in the lattice spacing) and this step is crucial for the success of the method. Note also that the one-loop correction is drastically smaller than the tree-level one, hinting at good convergence of this expansion. Moreover, the one-loop-corrected correlators are very close to the four-loop continuum perturbative curve [27], indicating that the remaining discretization effects are small at this lattice spacing.

In order to perform the continuum extrapolations, we need to follow the lines of constant physics. In our case, the only relevant scale is the correlator distance $x$, which we keep fixed in physical units by interpolating to the desired distance at all $\beta$ values. We use two interpolation ansatzes, linear and quadratic in $x^{2}$, between the two and three closest data points to find the interpolated value at each lattice spacing. We consider three lattice ("democratic") directions, for which hypercubic artifacts are known to be the smallest [24,25]: $\quad(0, k, k, k),-(k, k, k, k),-(0, k, k, 2 k)$ with $k \in\{1,2,3\}$ and interpolate independently for each of them. We note that other types of points do not make it possible to extract $\alpha_{s}$ at sufficiently small distances or break the rotational symmetry too severely ("nondemocratic" directions). Hence, similarly as in momentum-space studies of renormalization functions (see, e.g., Ref. [41]), the oneloop subtraction needs to be supplemented by a "democratic" criterion (see also the Supplemental Material). In this way, we keep the discretization effects related to the breaking of rotational symmetry well controlled and fixed as we change the lattice spacing. We use the difference of the two interpolation models as the systematic uncertainty associated to this step.

If discretization effects are under control, the continuum limits corresponding to the same physical distance should agree for each of the three lattice directions. We checked that this is the case and hence, we performed combined continuum fits of data for all three directions. Depending on the distance (and, thus, the available lattice spacings), we use from 6 to 12 data points and constrain the fit by a common value in the continuum, $C_{\Gamma}(1 / x, a=0)$. The fitting ansatz reads

$$
C_{\Gamma}(1 / x, a)=C_{\Gamma}(1 / x, a=0)+\sum_{i=\text { lattice direction }} \alpha_{i} a^{2}
$$

and has four fit parameters.

The difference between the axial and vector correlation functions was estimated in various frameworks, for a review see Ref. [42], including lattice QCD [21,43]. Also empirical data exist for this observable [44]. At short distances, the difference between the vector and axial correlators is reliably provided by the operator product expansion [45]. Using estimates from Ref. [46], the relative difference ranges from $0.03 \%$ at $x=0.1 \mathrm{fm}$ up to $1.5 \%$ at 


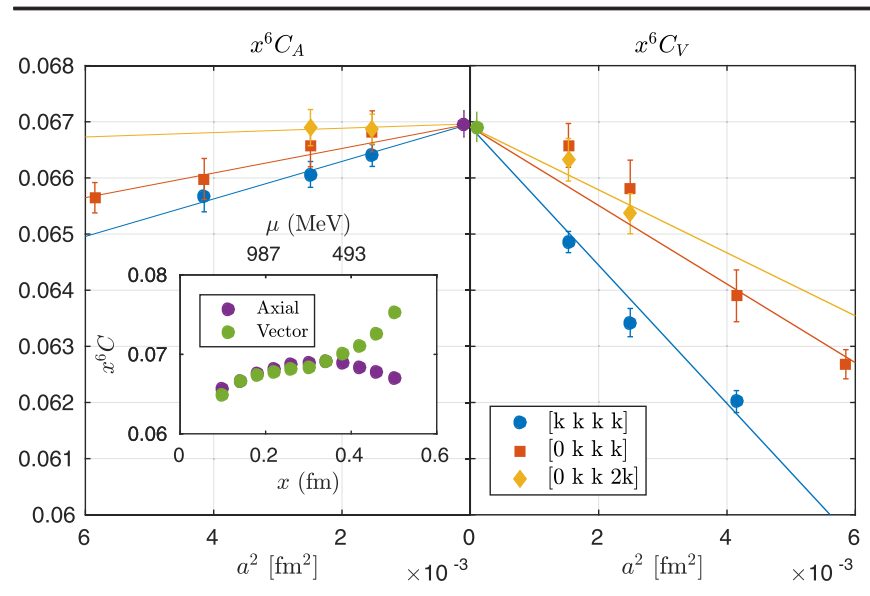

FIG. 2. Continuum extrapolation of the axial (left) and vector (right) correlators at $x=0.15 \mathrm{fm}$. Both extrapolations are performed independently, but their extrapolated values agree within uncertainties. The inset shows the distance dependence of the continuum-extrapolated axial and vector correlators. The error bars are smaller than the symbol size. Within our uncertainties, the correlators exhibit agreement for distances $\lesssim 0.35 \mathrm{fm}$.

$x=0.3 \mathrm{fm}$. Hence, within the statistical and systematic precision of our data, the two correlators are indistinguishable in that range of distances, see the inset of Fig. 2 and the Supplemental Material for more details. We use this observation in a two-fold way. (i) First, we employ it as a test of the reliability of the continuum extrapolation. In further analysis, we consider only the physical distances for which the independently extrapolated axial and vector correlators agree within their uncertainties. On the one hand, this criterion excludes lattice directions and physical distances which are too short and no control over discretization effects is possible, setting the lower limit to $0.1 \mathrm{fm}$. On the other hand, the two correlators are no longer equivalent at distances larger than around $0.35 \mathrm{fm}$ within our precision, which sets the upper limit on the physical distances where the impact of nonperturbative condensates is negligible. Note that the scale where the correlators become incompatible is related to effects of spontaneous chiral symmetry breaking and not to the breakdown of perturbation theory. In Fig. 2, we show an example of the continuum extrapolations of the axial and vector correlators at the physical distance of $x=0.15 \mathrm{fm}$. For examples for other distances, see the Supplemental Material. The fits are performed independently for both Dirac structures and in both cases, the combined fits to our three lattice directions provide a good description of the data, which holds also at other relevant distances (with $\chi^{2} /$ d.o.f. $\in[0.1,2]$ ). We emphasize that this is achieved only in the case of NSPT-corrected data, while continuum fits for only tree-level corrected data lead to $\chi^{2} /$ d.o.f. $\approx$ $10-20$ at the relevant distances. Moreover, although the individual data points at finite lattice spacing are different for different Dirac structures, $C_{A}=C_{V}$ in the continuum.

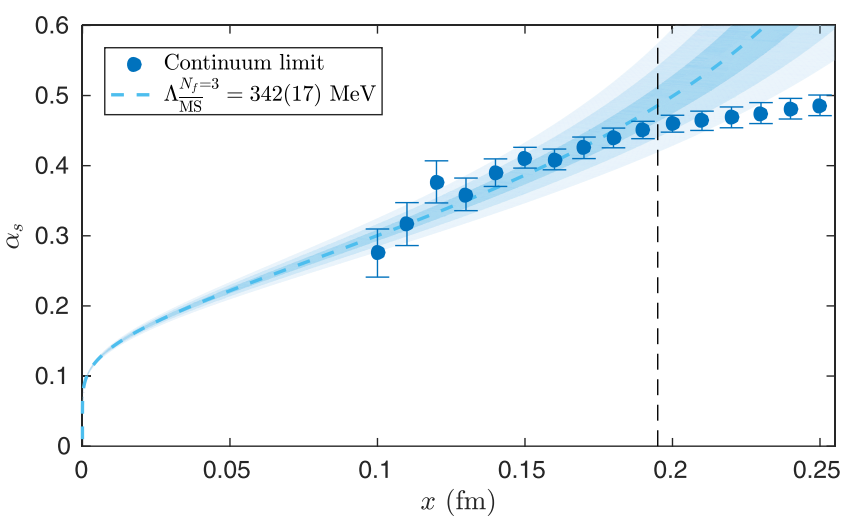

FIG. 3. Running of $\alpha_{s}$ extracted from the lattice data (blue points). At distances above around $0.2 \mathrm{fm}$, the running freezes and indicates the breakdown of matching to perturbation theory. The shaded blue band is the corresponding five-loop perturbative running [49-51] using the final value of $\Lambda_{\overline{\mathrm{MS}}}^{N_{f}}=3$ determined in this work (see section Final result). The darkest band corresponds to the $1-\sigma$ total uncertainty of $\Lambda_{\overline{\mathrm{MS}}}^{N_{f}=3}$ and the lighter bands to 2- $\sigma$ and $3-\sigma$.

(ii) Second, for the physical distances in the relevant range 0.1-0.2 fm, we use the independent data for $C_{A}$ and $C_{V}$ and consider their average, thus gaining in statistical precision.

Having the continuum-extrapolated $\overline{\mathrm{MS}}$ correlators, we know both sides of Eq. (2) and we can determine $\alpha_{\overline{\mathrm{MS}}}(\mu)$ for different scales, corresponding to different physical distances $1 / \mu=x$. The results are shown in Fig. 3. At distances above around $0.2 \mathrm{fm}$ (scales below $1 \mathrm{GeV}$ ), we observe that the running of the coupling freezes, indicating the breakdown of matching to four-loop perturbation theory. We convert our results for the coupling to $\Lambda_{\overline{\mathrm{MS}}}^{N_{f}}=3$ $[47,48]$ separately at each distance, see Fig. 4 . We show the perturbative running of $\alpha_{s}$ using our final value of the $\Lambda$ parameter in Fig. 3 and we discuss it below, after addressing systematic effects in our determination.

Final result.-We consider several sources of uncertainty in our analysis and we decompose the error of our final result for the $\Lambda$ parameter according to these different sources. The raw lattice correlators are, obviously, subject to statistical errors ("lat stat"). Extrapolating the correlators to the chiral limit has its associated systematic uncertainty ("chiral"). The perturbative subtraction of discretization effects via NSPT is also subject to statistical errors ("NSPT stat") and moreover, to a systematic uncertainty of extrapolation of NSPT results to the infinite volume limit ("NSPT infV"). The latter is computed as the difference between a polynomial fit to several volumes ranging from $V=32^{4}$ up to $V=80^{4}$ and the estimates from the largest volume $V=80^{4}$. The correlator interpolation uncertainty, described above, is denoted by "interpol." Renormalizing the correlators in the $\overline{\mathrm{MS}}$ scheme introduces an uncertainty from the values of $Z$ 


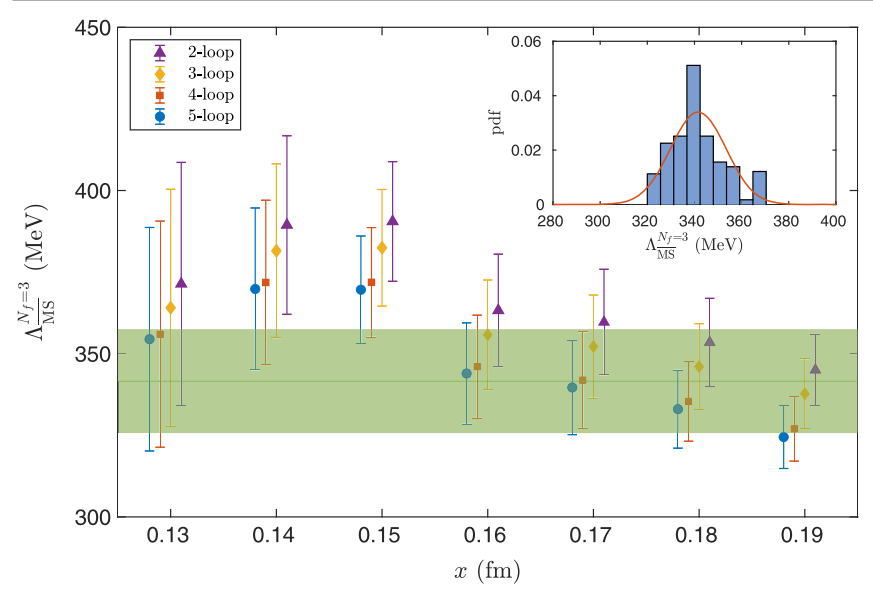

FIG. 4. Results for $\Lambda_{\frac{\mathrm{MS}}{N_{f}}=3}$ obtained from $\alpha_{s}$ at different distances. Shown are conversions between $\alpha_{s}$ and $\Lambda[47,48]$ using from two- to five-loop perturbative $\beta$ functions [49-51]. Our final value (indicated by the green band whose width is the total uncertainty) is obtained by a systematic procedure explained in the text. The inset shows the histogram of results corresponding to different ranges of distances taken into account in the systematic procedure, along with a Gaussian fit.

factors (" $Z_{A}$ " and " $Z_{V}$ "). The uncertainty of the $c_{V}$ and $c_{A}$ improvement coefficients is completely negligible compared to its other sources. Finally, we estimate the truncation uncertainty of the final $\Lambda$ value as the difference between conversions of $\alpha_{s}$ results to $\Lambda$ using the fourloop and five-loop $\beta$ functions ("trunc"). These differences are shown in Fig. 4, including also the twoloop and three-loop cases. The observed behavior suggests that while three-loop perturbation theory still shows significant truncation effects in the considered energy range, the four-loop and five-loop results evince convergence. We double this uncertainty to cover the truncation of the perturbative series of Eq. (2), where the five-loop coefficient is not available at present.

To make our final result independent from the choice of the window of physical distances where $\alpha_{s}$ is extracted, we adopt a systematic procedure similar to the one used in Ref. [25]. From all distances smaller than $0.2 \mathrm{fm}$, above which the coupling freezes, we choose the range 0.13$0.19 \mathrm{fm}$, where all other systematic uncertainties are under good control. Having seven determinations of $\Lambda$ corresponding to these different distances, we calculate all possible weighted averages covering from one to seven subsequent distances. We use the 28 resulting values of $\Lambda$ to build a weighted histogram, where the weights are taken as the squared inverse error of each individual result. The histogram is approximately Gaussian (see the inset of Fig. 4) and we fit its mean and width to determine the central value, i.e., $\Lambda_{\mathrm{MS}}^{N_{f}=3}$, and its uncertainty from the choice of the physical distances ("window"). This central value, along with the total uncertainty, is shown as the green band in Fig. 4.
The final result for the $\Lambda$ parameter reads

$$
\begin{aligned}
\Lambda_{\frac{\mathrm{MS}}{N_{f}=3}=} & 342(2.9)_{\mathrm{stat}}^{\mathrm{lat}}(5.0)_{\mathrm{chiral}}(6.5)_{\mathrm{stat}}^{\mathrm{NSPT}}(6.4)_{\mathrm{infV}}^{\mathrm{NSPT}}(0.8)_{Z_{A}} \\
& (1.0)_{Z_{V}}(0.4)_{\text {interpol }}(4.8)_{\text {trunc }}(12)_{\text {window }} \mathrm{MeV} \\
= & 342(17) \mathrm{MeV}
\end{aligned}
$$

where we combine the individual uncertainties in quadrature to obtain the total error. Our final value agrees well with earlier lattice determinations, e.g., with the recent one of Ref. [5], $\Lambda_{\overline{\mathrm{MS}}}^{N_{f}=3}=341(12) \mathrm{MeV}$, and with a comparable total error, dominated in our case by the uncertainty from the choice of the physical distance and by the uncertainty from the NSPT correction.

Discussion and conclusions. - In this Letter, we presented and tested a novel method to estimate the $\overline{\mathrm{MS}}$ strong coupling constant using numerical simulations of coordinate-space correlators and used it to determine the threeflavor QCD $\Lambda$ parameter. It is based on current-current correlation functions in position space at small distances. Our results suggest that the challenging multiscale problem of evaluating $\alpha_{s}$ can be addressed using lattices available today. We have shown that using a combination of state-ofthe-art simulations and novel analysis techniques, one can find a window of available scales $\mu$ and provide an estimate of $\Lambda_{\overline{\mathrm{MS}}}^{N_{f}=3}$ with competitive precision. In particular, the crucial steps are the perturbative subtraction of hypercubic artifacts and a combined continuum extrapolation using four lattice spacings and several lattice directions, which allowed us to control discretization effects at small distances in lattice units. We, furthermore, profited from independent evaluations of axial and vector correlators, which have a common continuum limit at short distances, to design a criterion to characterize the quality of continuum extrapolations and gain confidence in the results.

To conclude, we believe that the techniques described in this Letter provide a robust way of extracting the running of the QCD coupling and the QCD $\Lambda$ parameter, with good statistical precision and well-controlled sources of systematic effects. Furthermore, the precision reached in this work can be increased even more in a systematic way. Techniques based on current-current correlators in position space, improved by NSPT reduction of discretization effects, can be useful to determine other quantities, such as the quark condensate.

We gratefully acknowledge discussions with V. Braun, F. Knechtli, and T. Korzec. This research was carried out with the support of the Interdisciplinary Centre for Mathematical and Computational Modelling (ICM) University of Warsaw under Grants No. GA67-12, No. GA69-20, No. GA71-26, No. GA76-14, and AGH Cyfronet Computing Center under Grant ID pionda, nspt, hadronspectrum. This work was supported by Deutsche Forschungsgemeinschaft under Grant No. SFB/TRR 55 and in part by the Polish 
Narodowe Centrum Nauki (NCN) Grants No. UMO-2016/ 21/B/ST2/01492 (P. K. and S. C.) and No. 2016/22/E/ST2/ 00013 (K. C.). P. K. acknowledges support from the Narodowa Agencja Wymiany Akademickiej (NAWA) Bekker fellowship and thanks Università degli Studi di Roma "Tor Vergata" for hospitality during which this work was initiated. We thank our colleagues in the Coordinated Lattice Simulations (CLS) effort for the joint generation of the gauge field ensembles on which the computation described here is based. The gauge ensembles were generated with the help of the Gauss Centre for Supercomputing e.V. using computer time allocations on SuperMUC at Leibniz Supercomputing Centre (LRZ) and JUQUEEN at Jülich Supercomputing Center (JSC). GCS is the alliance of the three national supercomputing centers HLRS (Universität Stuttgart), JSC (Forschungszentrum Jülich) and LRZ (Bayerische Akademie der Wissenschaften), funded by the German Federal Ministry of Education and Research (BMBF) and the German State Ministries for Research of Baden-Württemberg (MWK), Bayern (StMWFK) and Nordrhein-Westfalen (MIWF). Additionally computer time provided by PRACE (Partnership for Advanced Computing in Europe) as part of the project ContQCD was used. Additional ensembles were generated on QPACE2 supercomputer at University of Regensburg and on the Wilson computer cluster at University of Mainz. For the NSPT computer resources of QPACE3 at the Juelich Supercomputing Center were used.

*salvatore.cali@uj.edu.pl

†krzysztof.cichy@gmail.com

"piotr.korcyl@uj.edu.pl

jakob.simeth@ur.de

[1] G. P. Salam, in From My Vast Repertoire ...: Guido Altarelli's Legacy, edited by A. Levy, S. Forte, and G. Ridolfi (World Scientific Publishing, Singapore, 2019), pp. 101-121 [arXiv:1712.05165].

[2] D. J. Gross and F. Wilczek, Phys. Rev. Lett. 30, 1343 (1973).

[3] H. D. Politzer, Phys. Rev. Lett. 30, 1346 (1973).

[4] M. Tanabashi et al. (Particle Data Group), Phys. Rev. D 98, 030001 (2018).

[5] M. Bruno, M. D. Brida, P. Fritzsch, T. Korzec, A. Ramos, S. Schaefer, H. Simma, S. Sint, and R. Sommer (ALPHA Collaboration), Phys. Rev. Lett. 119, 102001 (2017).

[6] K.-I. Ishikawa, I. Kanamori, Y. Murakami, A. Nakamura, M. Okawa, and R. Ueno, J. High Energy Phys. 12 (2017) 067.

[7] N. Husung, M. Koren, P. Krah, and R. Sommer, in Proceedings, 35th International Symposium on Lattice Field Theory (Lattice 2017): Granada, Spain, 2017; EPJ Web Conf. 175, 14024 (2018).

[8] F. Karbstein, M. Wagner, and M. Weber, Phys. Rev. D 98, 114506 (2018).
[9] H. Takaura, T. Kaneko, Y. Kiyo, and Y. Sumino, Phys. Lett. B 789, 598 (2019).

[10] R. J. Hudspith, R. Lewis, K. Maltman, and E. Shintani, arXiv:1804.10286.

[11] Y. Maezawa and P. Petreczky, Phys. Rev. D 94, 034507 (2016).

[12] S. Zafeiropoulos, P. Boucaud, F. De Soto, J. RodríguezQuintero, and J. Segovia, Phys. Rev. Lett. 122, 162002 (2019).

[13] K. Nakayama, H. Fukaya, and S. Hashimoto, Phys. Rev. D 98, 014501 (2018).

[14] S. Aoki et al. (Flavour Lattice Averaging Group), Eur. Phys. J. C 80, 113 (2020).

[15] M. Bruno et al. (CLS Collaboration), J. High Energy Phys. 02 (2015) 043.

[16] G. S. Bali, E. E. Scholz, J. Simeth, and W. Söldner (RQCD Collaboration), Phys. Rev. D 94, 074501 (2016).

[17] M. Dalla Brida, T. Korzec, S. Sint, and P. Vilaseca, Eur. Phys. J. C 79, 23 (2019).

[18] J. Bulava, M. Della Morte, J. Heitger, and C. Wittemeier (ALPHA Collaboration), Nucl. Phys. B896, 555 (2015).

[19] J. Heitger, F. Joswig, A. Vladikas, and C. Wittemeier, in Proceedings, 35th International Symposium on Lattice Field Theory (Lattice 2017): Granada, Spain, 2017; EPJ Web Conf. 175, 10004 (2018).

[20] F. Di Renzo and L. Scorzato, J. High Energy Phys. 10 (2004) 073.

[21] M. Tomii, G. Cossu, B. Fahy, H. Fukaya, S. Hashimoto, T. Kaneko, and J. Noaki (JLQCD Collaboration), Phys. Rev. D 96, 054511 (2017).

[22] M. Tomii and N. H. Christ, Phys. Rev. D 99, 014515 (2019).

[23] V. Gimenez, L. Giusti, S. Guerriero, V. Lubicz, G. Martinelli, S. Petrarca, J. Reyes, B. Taglienti, and E. Trevigne, Phys. Lett. B 598, 227 (2004).

[24] K. Cichy, K. Jansen, and P. Korcyl, Nucl. Phys. B865, 268 (2012).

[25] K. Cichy, K. Jansen, and P. Korcyl, Nucl. Phys. B913, 278 (2016).

[26] M. Tomii, G. Cossu, B. Fahy, H. Fukaya, S. Hashimoto, T. Kaneko, and J. Noaki (JLQCD Collaboration), Phys. Rev. D 94, 054504 (2016).

[27] K. G. Chetyrkin and A. Maier, Nucl. Phys. B844, 266 (2011).

[28] G. S. Bali, S. Collins, and A. Schafer, Comput. Phys. Commun. 181, 1570 (2010).

[29] G. S. Bali, S. Collins, P. Georg, B. Gläßle, P. Korcyl, A. Rabenstein, D. Richtmann, A. Schäfer, E. E. Scholz, J. Simeth, W. Söldner, and P. Wein (RQCD Collaboration) (to be published).

[30] M. Lüscher, J. High Energy Phys. 08 (2010) 071; 03 (2014) 092(E).

[31] See Supplemental Material at http://link.aps.org/ supplemental/10.1103/PhysRevLett.125.242002 for further details, which also includes Refs. [32-35].

[32] C. Torrero and G. S. Bali, Proc. Sci., LATTICE2008 (2008) 215 [arXiv:0812.1680].

[33] G. S. Bali, C. Bauer, A. Pineda, and C. Torrero, Phys. Rev. D 87, 094517 (2013).

[34] H. Panagopoulos and Y. Proestos, Phys. Rev. D 65, 014511 (2001). 
[35] M. Davier, A. Höcker, B. Malaescu, C.-Z. Yuan, and Z. Zhang, Eur. Phys. J. C 74, 2803 (2014).

[36] S. Sint, Nucl. Phys. B847, 491 (2011).

[37] J. Simeth, A. Sternbeck, E.-M. Ilgenfritz, H. Perlt, and A. Schiller, Proc. Sci., LATTICE2013 (2014) 459 [arXiv:1311 .1934].

[38] J. Simeth, A. Sternbeck, M. Göckeler, H. Perlt, and A. Schiller, Proc. Sci., LATTICE2014 (2015) 294 [arXiv:1501 .06322].

[39] Y. Taniguchi and A. Ukawa, Phys. Rev. D 58, 114503 (1998).

[40] P. Korcyl and J. Simeth (to be published).

[41] C. Alexandrou, M. Constantinou, and H. Panagopoulos (ETM Collaboration), Phys. Rev. D 95, 034505 (2017).

[42] E. V. Shuryak, Rev. Mod. Phys. 65, 1 (1993).

[43] T. DeGrand, Phys. Rev. D 64, 094508 (2001).
[44] R. Barate et al. (ALEPH Collaboration), Eur. Phys. J. C 4, 409 (1998).

[45] M. Shifman, A. Vainshtein, and V. Zakharov, Nucl. Phys. B147, 385 (1979).

[46] T. Schäfer and E. V. Shuryak, Phys. Rev. Lett. 86, 3973 (2001).

[47] C. G. Callan, Jr., Phys. Rev. D 2, 1541 (1970).

[48] K. Symanzik, Commun. Math. Phys. 18, 227 (1970).

[49] P. A. Baikov, K. G. Chetyrkin, and J. H. Kühn, Phys. Rev. Lett. 118, 082002 (2017).

[50] T. Luthe, A. Maier, P. Marquard, and Y. Schröder, J. High Energy Phys. 07 (2016) 127.

[51] F. Herzog, B. Ruijl, T. Ueda, J. A. M. Vermaseren, and A. Vogt, J. High Energy Phys. 02 (2017) 090. 